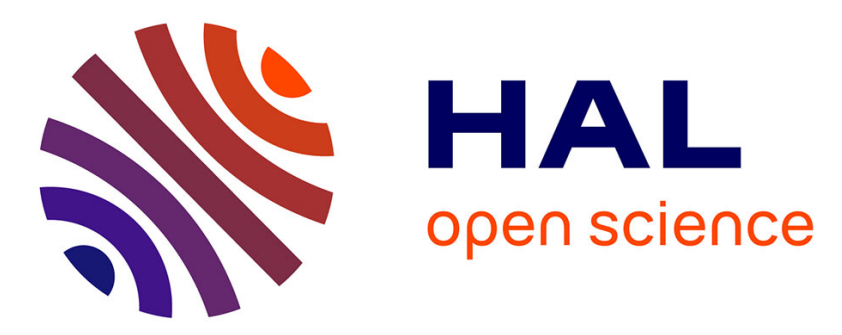

\title{
A hardware efficient 3-bit second-order dynamic element matching circuit clocked at $300 \mathrm{MHz}$
}

Esmaeil Najafi Aghdam, Philippe Benabes

\section{To cite this version:}

Esmaeil Najafi Aghdam, Philippe Benabes. A hardware efficient 3-bit second-order dynamic element matching circuit clocked at 300MHz. International Symposium on Circuits and Systems, May 2006, Island of Kos, Greece. pp.2977-2980, 10.1109/ISCAS.2006.1693250 . hal-00257788

\section{HAL Id: hal-00257788 \\ https://hal-centralesupelec.archives-ouvertes.fr/hal-00257788}

Submitted on 20 Feb 2008

HAL is a multi-disciplinary open access archive for the deposit and dissemination of scientific research documents, whether they are published or not. The documents may come from teaching and research institutions in France or abroad, or from public or private research centers.
L'archive ouverte pluridisciplinaire HAL, est destinée au dépôt et à la diffusion de documents scientifiques de niveau recherche, publiés ou non, émanant des établissements d'enseignement et de recherche français ou étrangers, des laboratoires publics ou privés. 


\section{A hardware efficient 3-bit second-order dynamic element matching circuit clocked at $300 \mathrm{MHz}$}

Esmaeil Najafi Aghdam, Esmaeil.Aghdam@supelec.fr, and Philippe Benabes, Philippe.Benabes@supelec.fr Dept. of Signal Processing and Electronics Systems, SUPELEC, P. de Moulon, Gif sur Yvette, F-91192, Franc.

\begin{abstract}
A robust and hardware efficient dynamic element matching $(D E M)$ algorithm is developed and used to design a $4^{t h}$-order bandpass $(B P)$ mismatch-shaping circuit, moved inside the feedback loop of a $6^{\text {th }}$-order bandpass continuous-time delta-sigma modulator. This algorithm is based on a shortened tree-structured scheme $(S T D E M)$ which can assure a stable high order mismatch-shaping with a modest circuit volume. The modulator has a 3-bit quantizer and 8 thermometric feedback $D A C$ 's cells. The designed DEM's circuits is simulated in $\mathbf{0 . 3 5} \mu$ CMOS which can be clocked up to $300-\mathrm{MHz}$. The mismatch error floor is decreased of about $35 \mathrm{~dB}$ in the band of interest. Its related circuit occupies of about $0.22 \mathrm{~mm}^{2}$ area.
\end{abstract}

\section{INTRODUCTION}

With increasing demand of $\Delta \Sigma$ modulators $(D S M \mathrm{~s})$ with broader bandwidth and wider dynamic range $(D R)$, multibit architectures become attractive for this trend as [1], [2]:

- the $S N R$ directly increases by $6 d B$ for each extra quantization bit, resulting in lower OSR application possible,

- multibit DSM's loop possesses better stability resulting in additional loop gain for higher order structure, which in turn results indirectly in improved $S N R$,

- it is one of the best ways to reduce clock jitter noise resulting in high frequency application possible,

- it possesses lower idle tone and lower out of band noise,

- in multibit DSM, the first opamp needs lower input range and slew-rate resulting in lower power consumption.

On the other hand, a multibit DSM needs a multibit-DAC on the feedback path which is usually a thermometric current steering $D A C$ limited to 5-bits. Any feedback-DAC can suffer from inevitable mismatching occurred during fabrication process. This is a large disadvantage of the multibit DAC which seriously degrades its $S N R$, as it acts in the feedback path. Multibit architecture has no other sever inconvenience and its circuits' complexity can be accepted if one needs such many advantages mentioned above.

In order to integrate a multibit DSM, several error correction methods have been developed as trimming, calibration, digital correction, and dynamic element matching (DEM). The last one is widely used in high performance integrated modulators having a resolution over 10 bits. This technique can be realized in different ways. Randomization scheme whitens DAC's mismatch errors over whole frequency range, so that input depended tones are diminished but its noise floor increases in the band of interest. Thus, better solution can be using a mismatch noise shaping technique. The well-known data weighted averaging method $(D W A)$ can effectively be used

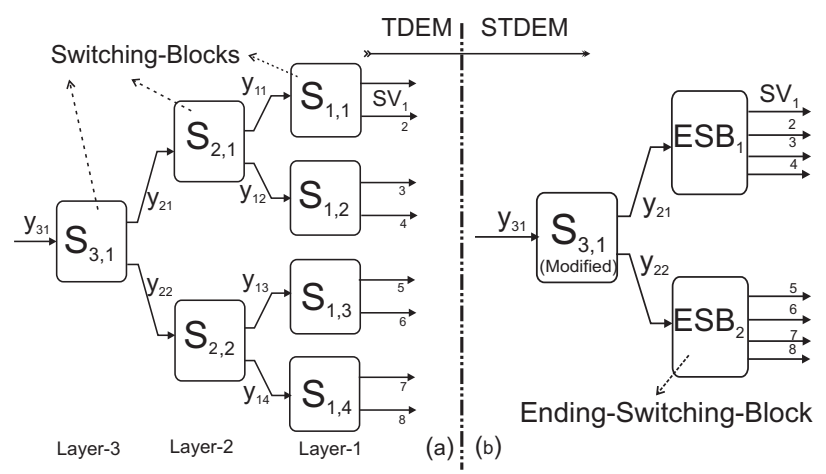

Fig. 1. Dynamic element matching algorithm: a)TDEM, b)STDEM

to shape mismatch errors reside in signal band. However, with the same frequency as in the quantizer, it can mainly be applied as a first order lowpass mismatch-shaping. For higher order mismatch-shaping error, only two original methods have been introduced; feedback-vector or sorting algorithm (SDEM) [3] and tree-structured scheme (TDEM) [4]. The SDEM suffers from lower hardware efficiency and clock rate limits, especially for higher number of quantization level. The TDEM suffers more from algorithm instability for high order mismatch-shaping.

The authors have lately developed two new schemes, which are based on two mentioned original methods. The first one, called $M D E M$, is a mixed structured of SDEM and TDEM [5]. The MDEM benefits of better stability nature of SDEM and hardware efficiency of TDEM. The second one, called $S T D E M$, is a shortened tree-structured introduced in [6]. It is more stable than the pure TDEM with the same hardware efficiency. This paper tends to further illustrate the STDEM algorithm and introduces its related circuits, which are designed for a 3-bit feedback-DAC, in two next sections.

\section{Shortened TREe-STRUCTURE DEM ( STDEM)}

STDEM algorithm is based on conventional TDEM [4], [6]. Fig.1 shows an example of a 9-level TDEM (on the left) and its equivalent STDEM (on the right). These two algorithms have two main differences, which results in a better performance for STDEM. Each group of the last three blocks in TDEM structure is replaced by an Ending Switching Block (ESB) to obtain a STDEM structure. In addition, the remaining switching blocks in STDEM will act in different way from that of TDEM. Some more details of STDEM will be developed here in the below.

Generally, a $\left(1+2^{B}\right)$-level TDEM consists of $B$ layers shown 
as $B$ different columns (see a 3-bit example in Fig.1-a). Each $k^{t h}$-layer can also consist of $2^{(B-k)}$ boxes laid out in rows. All boxes within the tree structure are called switching blocks $(S B)$, are labeled $S_{k, r}$, where $k$ denotes the layer number and $r$ denotes its position in the layer. Each $S_{k, r}$ has a $(k+1)$-bit input $y_{k r}$ and two $k$-bit output: $y_{k-1,2 r-1}=\left(y_{k r}+S_{k r}\right) / 2$ and $y_{k-1,2 r}=\left(y_{k r}-S_{k r}\right) / 2$. Also, $S_{k r}(n)$ must satisfy certain conditions for number conservation rule, as:

$$
\begin{aligned}
S_{k r}(n) & =\left\{\begin{array}{c}
\text { even if } y_{k r} \text { is even } \\
\text { odd if } y_{k r} \text { is odd }
\end{array}\right. \\
\left|S_{k r}(n)\right| & \leq \min \left\{y_{k r}(n), 2^{k}-y_{k r}(n)\right\}
\end{aligned}
$$

Its suitable structure is shown in Fig.2. The special quantizer
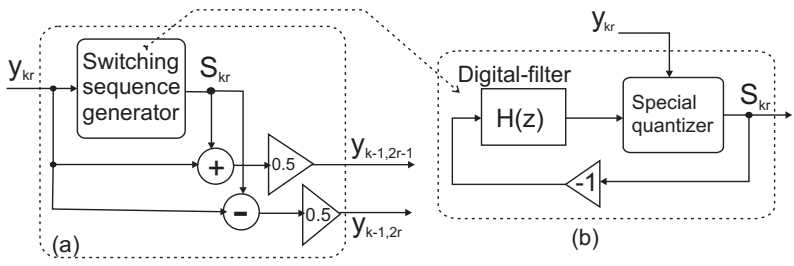

Fig. 2. a)Conventional SB's structure b)switching sequence generator

transfer function was first defined as:

$$
S_{k r}(n)=\left\{\begin{array}{cc}
1 & \text { if } y_{k r} \text { is odd and } V_{k r}>0 \\
-1 & \text { if } y_{k r} \text { is odd and } V_{k r}<0 \\
0 & \text { in all other cases }
\end{array}\right.
$$

This definition imposes a very strict rule to produce $S_{k r}(n)$ sequences so that it takes only zero for any even SB's inputs. This in turn, can cause instability in the mismatch shaping loop and overflow can occurre in the second and following stages of the filter if the input of the $S B$ is even for a few periods. To meet better stability, it was then modified [7]:

$$
S_{k r}(n)=\left\{\begin{array}{cc}
+1 & y_{k r} \text { is odd and } V_{k r}>0 \\
-1 & y_{k r} \text { is odd and } V_{k r}<0 \\
+2 & y_{k r} \text { is divisible by } 4 \text { and } V_{k r}>0 \\
-2 & y_{k r} \text { is divisible by } 4 \text { and } V_{k r}<0 \\
0 & \text { in all other cases }
\end{array}\right.
$$

The above-mentioned modification results in better stability, but it is not sufficient and a further restriction must be added to maintain at least a first order mismatch shaping functioning until the related SB comes out of its unstable situation [7]. On the other hand, Eq.3 is only applicable until the second layer. This is because the two first layers' inputs can only lie between $[0,4]$ thus, Eq.3 recalls its origin from Eq.2. In conclusion, TDEM algorithm can hardly handle a pure simple second or higher order DEM. In [5], a mixed algorithm (MDEM) solved this problem by replacing a partially SDEM. It seemed to be a good idea but at the price of additional logic.

Recently, a hardware efficient $S T D E M$ solution was presented in [6] in which the first layer is completely eliminated, as shown in Fig.1.b. Here, the tree-structure DEM algorithm is well suited if there are no more than 4-DAC cells to control. Then, an ESB, which consists of three independent digital filters and one decision logic, controls each group of 4-DACunits (see Fig.3). Digital filters in the ESB can be a cascade of some (usually 2 or 3 ) integrators for lowpass and some

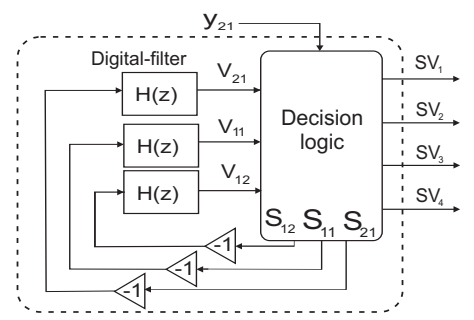

Fig. 3. General structure of an ESB used in STDEM algorithm.

resonators for bandpass applications. The decision logic is based on table-I, wherein the priority of any assignment for $S_{i j}, i, j \in\{1,2\}$ is forced to regulate critical volume in digital filters in the related ESB. Threshold levels $t_{2}$ and $t_{1}$ are depended on the filter's order and structure which can be optimized by simulation or estimation. In order to fulfill number conservation rule and to be compatible with the rest of global TDEM, table-I must obey following expressions:

$$
\begin{array}{lll}
S_{11}=s v_{1}-s v_{2}, & S_{21}=s v_{1}+s v_{2}-\left(s v_{3}+s v_{4}\right) \\
S_{12}=s v_{3}-s v_{4}, & y_{21}=s v_{1}+s v_{2}+s v_{3}+s v_{4}
\end{array}
$$

Furthermore, to neutralize the input dependence at the following layers the special quantizer transfer functions of all the remained SBs are replaced by:

$$
S_{k r}(n)=\left\{\begin{array}{ccc}
+1 & y_{k r} & \text { odd and } V_{k r}>0 \\
-1 & y_{k r} & \text { odd and } V_{k r}<0 \\
+2 & 0<y_{k r}<2^{k} & \text { even and } V_{k r}>0 \\
-2 & 0<y_{k r}<2^{k} & \text { even and } V_{k r}<0 \\
0 & \text { in all } & \text { other cases }
\end{array}\right.
$$

By this mean, there is no serious instability problem in switching blocks any more since $S_{k r}(n)$ accepts a desired non-zero value for any non zero input to the related SB. The circumstances of this replacement were analyzed and a mathematical expression for a $b$-bit STDEM-DAC's analog output $D o_{b}(n)$ is formulated in [6]. It is similar to that of TDEM in [4] as:

$$
\begin{aligned}
D o_{B}(n) & =(1+\bar{\alpha}) y_{B}(n)+e(n)+\epsilon \\
\bar{\alpha} & =\frac{1}{2^{B}} \sum_{i=1}^{2^{B}} \alpha_{i}, \text { and } \epsilon=\sum_{i=1}^{2^{B}} \epsilon_{i} \\
e(n) & =\sum_{k=1}^{B} \sum_{r=1}^{2^{B-k}} \Delta_{k r} S_{k r}(n) \\
\Delta_{k r} & =\frac{1}{2^{k}} \sum_{i=(r-1) 2^{k}+2^{k-1}}^{(r-1)} \alpha_{i}-\alpha_{i+2^{k-1}}
\end{aligned}
$$

where $\bar{\alpha}$ is a gain error and affects only the loop gain, $\epsilon$ is an offset error and $e(n)$ is an input depended nonlinearity error. The offset error $\epsilon$ cannot be corrected by DEM methods and normally does not have a destructive effect. The most important error is $e(n)$ which must be reduced towards zero in the band of interest. Regarding $e(n)$ 's equation, all of the $S_{k r}(n)$ must be a vector with a desired shape through different digital filters. For example, a 3-bit feedback DAC mismatch-shaping system needs 7 digital filters feeding by 7 shaped-sequences $\left\{S_{31}, S_{21}, S_{11}, S_{12}, S_{21}^{\prime}, S_{11}^{\prime}, S_{12}^{\prime}\right\}$, in both TDEM and STDEM algorithms. However, in STDEM, these sequences can almost freely accept nonzero values even if they 
TABLE I

DECISION LOGIC IN THE ESB

\begin{tabular}{|c|c|c|c|}
\hline$Y_{21}$ & Conditions on: $\mathrm{v}_{21}, \mathrm{v}_{11}, \mathrm{v}_{12}$ & $s_{21} s_{11}, s_{12}$ & $s v 1-4$ \\
\hline 0 & Don't-care & $0,0,0$ & 0000 \\
\hline 1 & $\mathrm{v}_{21} \geq-\mathrm{t}_{2}, \mathrm{v}_{11} \geq \mathrm{t}_{1}$ & $1,1,0$ & 1000 \\
\hline$"$ & $\mathrm{v}_{21} \geq 0, \mathrm{v}_{11} \geq 0,-\mathrm{t}_{1} \leq \mathrm{v}_{12}<t_{1}$ & $1,1,0$ & 1000 \\
\hline$"$ & $\mathrm{v}_{21}<t_{2}, \mathrm{v}_{11}<-t_{1}$ & $1,-1,0$ & 0100 \\
\hline$"$ & $\mathrm{v}_{21} \geq 0, \mathrm{v}_{11}<0,-\mathrm{t}_{1} \leq \mathrm{v}_{12}<t_{1}$ & $1,-1,0$ & 0100 \\
\hline$"$ & $-\mathrm{t}_{1} \leq \mathrm{v}_{11}<t_{1}, \mathrm{v}_{12} \geq \mathrm{t}_{1}$ & $-1,0,1$ & 0010 \\
\hline$"$ & $\mathrm{~V}_{21}<0,-\mathrm{t}_{1} \leq \mathrm{V}_{11}<t_{1}, \mathrm{~V}_{12} \geq 0$ & $-1,0,1$ & 0010 \\
\hline$"$ & $-\mathrm{t}_{1} \leq \mathrm{v}_{11}<t_{1}, \mathrm{v}_{12}<-t_{1}$ & $-1,0,-1$ & 0001 \\
\hline$"$ & $\mathrm{v}_{21}<0,-\mathrm{t}_{1} \leq \mathrm{v}_{11}<t_{1}, \mathrm{v}_{12}<0$ & $-1,0,-1$ & 0001 \\
\hline 2 & $\mathrm{v}_{21} \geq \mathrm{t}_{2}$ & $2,0,0$ & 1100 \\
\hline$"$ & $\mathrm{v}_{21}<-t_{2}$ & $-2,0,0$ & 0011 \\
\hline$"$ & $-\mathrm{t}_{2} \leq \mathrm{v}_{21}<t_{2}, \mathrm{v}_{11} \geq 0, \mathrm{v}_{12} \geq 0$ & $0,1,1$ & 1010 \\
\hline " & $-\mathrm{t}_{2} \leq \mathrm{v}_{21}<t_{2}, \mathrm{v}_{11} \geq 0, \mathrm{v}_{12}<0$ & $0,1,-1$ & 1001 \\
\hline$"$ & $-\mathrm{t}_{2} \leq \mathrm{v}_{21}<t_{2}, \mathrm{v}_{11}<0, \mathrm{v}_{12} \geq 0$ & $0,-1,1$ & 0110 \\
\hline$"$ & $-\mathrm{t}_{2} \leq \mathrm{v}_{21}<t_{2}, \mathrm{v}_{11}<0, \mathrm{v}_{12}<0$ & $0,-1,-1$ & 0101 \\
\hline 3 & $-\mathrm{t}_{1} \leq \mathrm{v}_{11}<t_{1}, \mathrm{v}_{12} \geq \mathrm{t}_{1}$ & $1,0,1$ & 1110 \\
\hline$"$ & $\mathrm{v}_{21} \geq 0,-\mathrm{t}_{1} \leq \mathrm{v}_{11}<t_{1}, \mathrm{v}_{12} \geq 0$ & $1,0,1$ & 1110 \\
\hline$"$ & $-\mathrm{t}_{1} \leq \mathrm{V}_{11}<t_{1}, \mathrm{v}_{12}<-t_{1}$ & $1,0,-1$ & 1101 \\
\hline$"$ & $\mathrm{~V}_{21} \geq 0,-\mathrm{t}_{1} \leq \mathrm{V}_{11}<t_{1}, \mathrm{~V}_{12}<0$ & $1,0,-1$ & 1101 \\
\hline " & $\mathrm{v}_{11} \geq \mathrm{t}_{1}$ & $-1,1,0$ & 1011 \\
\hline$"$ & $\mathrm{v}_{21}<0, \mathrm{v}_{11} \geq 0,-\mathrm{t}_{1} \leq \mathrm{v}_{12}<t_{1}$ & $-1,1,0$ & 1011 \\
\hline$"$ & $\mathrm{v}_{11}<-t_{1}$ & $-1,-1,0$ & 0111 \\
\hline$"$ & $\mathrm{~V}_{21}<0, \mathrm{~V}_{11}<0,-\mathrm{t}_{1} \leq \mathrm{V}_{12}<t_{1}$ & $-1,-1,0$ & 0111 \\
\hline 4 & Don't-care & $0,0,0$ & 1111 \\
\hline
\end{tabular}

receive even inputs. In fact, such a sequence has a much better mismatch-shaping performance than that of a conventional TDEM, which is realized by merging two first layers and using dynamic decision rules in its true table as well as employing Eq.5 in the other layers.

\section{CiRCUIT DESCRIPTION AND SIMUlation RESUltS}

In today's CMOS technologies, circuits' mismatch standard deviation is in the order of a few tenth percents, (usually $0.1 \% \leq \delta I \leq 1 \%$ ). This level of mismatch causes noise floor and unwanted tones to be increased in the band of interest. Without any correction, the worst-case standard deviation of the normalized DAC-output for a B-bit thermometric structure can be expressed as [2]:

$$
\delta\left[D A C_{\text {out }, d B}\right]=20 \log \left[\frac{\delta I}{2 \sqrt{2^{B}+1}}\right]
$$

For example, supposing, $\delta I=0.01$ and $B=3$ results in a distortion of $\approx-70 d B$. In order to suppress this mismatchnoise in a high-resolution DSM (usually $D R \geq 90 \mathrm{~dB}$ ), using a first order noise shaping is not sufficient. Thus, a $2^{d}$-order lowpass or a $4^{\text {th }}$-order BP mismatch-shaping system will be needed regarding our applications ${ }^{1}$.

Fig.4 shows a modified switching-block diagram for a $4^{\text {th }}$ order BP-STDEM. It is completely realized in the digital domain. Since output of the special quantizer in such a modified SB's loop can accept a non-zero value for all of its non-zero inputs, the register's resolution requirement is quite modest, maximum five-bit at last resonator $\left(V_{31}\right)$.

Fig. 5 shows the present design of the $4^{t h}$ order bandpass ESB wherein a sufficiently fluid group of decision rules is based on equations-4 and table-I. In order to estimate threshold levels

\footnotetext{
${ }^{1}$ Note that a fourth-order bandpass noise-shaping centered at $f_{s} / 4$ is equivalent to that of a similar second order lowpass one
}

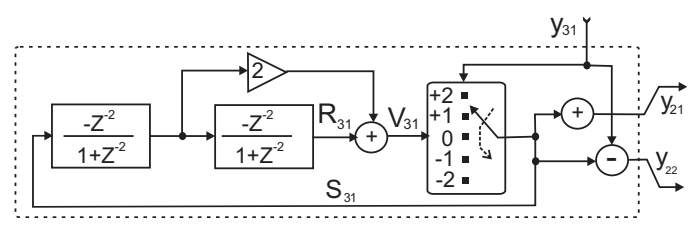

Fig. 4. Modified SB, used in all layers in STDEM but the last-one

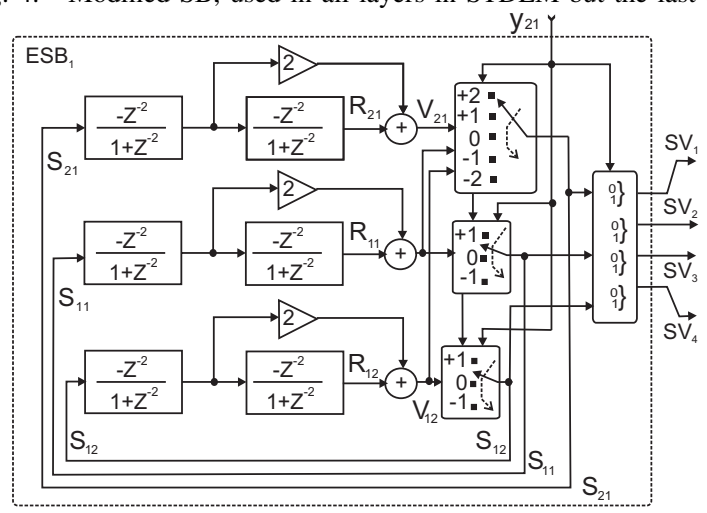

Fig. 5. Ending switching block schematic, used in the last layer of STDEM

$t_{1}$ and $t_{2}$, the critical volumes of digital filters' output values have to be considered. For the loop containing $S_{21}, R_{21}$ and $V_{21}$, such a critical situation will start when the loop cannot be controlled for at least two periods $\left(S_{21}=0\right)$. If loop's integrators (or resonators) have non-zero values just before receiving such a tail of zero $S_{21}$, the second integrator can be overflowed in some periods. For example, when integrators' initial outputs are supposed to be 4 then, first one remains unchanged but the second stage output (here $R_{21}$ ) becomes grater than 8 and $V_{21} \geq 16$, while $S_{21}$ is still zero. Therefore, the threshold value $t_{1}$ has to detect such an output growing tendency. This can simply be realized by regarding present value of $V_{21}$ as in table-I. The estimated threshold level with this simple example is in the order of 16 and -16, respectively for positive and negative resonator's output. However, we are naturally interested in its minimum possible value to strictly control registers' values. Thus, in the same reasoning way but for one period of an unwanted output growing, a lowest threshold level estimated of about 8. In different practical simulations, these estimated values quietly results in proper mismatch shaping. The optimum value is obtained between 8 and 12, which is theoretically expected before. We can estimate the value of the second threshold level $t_{2, \text { opt }}$ in the same manner. The estimated $t_{2}$ value is 8 . Its optimized value is 6 . However, there is no much difference if it is practically considered $t_{2, o p t}=8$. This method can also be used to estimate maximum resolution of the registers. If we suppose that such a critical situation can be present for about four further periods, the second register output $\left(R_{21_{\text {out }}}\right)$ can reach up to 28 which needs 5-bit resolution. This is well confirmed by different simulation, so that it rarely exceeds over 20 . In order to give a better sense of this judgment, Fig.6 shows the registers' output levels versus different DSM-input levels, where the same output of a conventional TDEM algorithm is depicted to be compared. Even-if one accepts using such a long register resolution, the mismatch-shaping action does 
not occur properly. This phenomenon depends on the system's OSR so that DAC-mismatch errors can only be shaped if the related loop is controlled faster than the signal transition. In digital frequency domain, it is represented by a very narrow band around central frequency as depicted in Fig.7-a for a conventional TDEM, which is improved in Fig.7-b by STDEM method.

Also, register's maximum output level in a modified-SB is

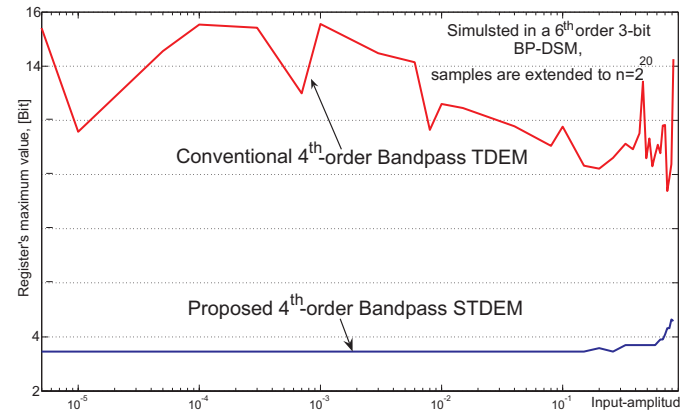

Fig. 6. Registers' output levels [bit], in a ESB and conventional TDEM

usually lower than that of ESB. This is because its special quantizer based on equation- 5 is not limited by the input types (odd or even). In addition, they have more chances to receive a non-zero input since they process any input at first.

The STDEM's designed circuits are simulated at system level with fixed register's precision. As discussed in the previous section, all registers' resolutions are limited to 5-bits plus a sign-bit. Fig.8 shows the SNR versus input level for a $6^{\text {th }}$ order bandpass DSM with a 3-bit quantizer [8]. A given mismatch error level of $\delta I=0.01$ is properly corrected by a $4^{\text {th }}$ STDEM, which follows the ideal case. However, using $4^{\text {th }}$-order conventional bandpass-TDEMs does not enhance the system's performance and its $S N R$ may even stay below that of a pure system without any DEM. The mentioned STDEM has been implemented in $C A D E N C E$ environment using $V E R I L O G$, then optimized by its $A M B I T$ software tools. Fig.9 shows modulator output spectrum for ideal, without any mismatch shaping and mismatch-shaping using a $4^{\text {th }}$-order bandpass STDEM when DAC's cells have 0.9\% mismatch. The mismatch noise floor is decreased by ca. $35 d B$ in the band of interest for $O S R=100$. This circuit can be clocked with a maximum rate of $300 \mathrm{MHz}$ if it is implemented in a $0.35 \mu \mathrm{m}$-CMOS technology. In order to use the presented STDEM in a high-speed DSM, one period delay must be considered and compensated in system levels [9]. It needs a $0.22 \mu \mathrm{m}^{2}$ area and contains about 3.000 gates as reported by AMBit. In comparison, a similar SDEM needs $0.6 \mu m^{2}$ area which can be clocked twice slower than a similar STDEM.
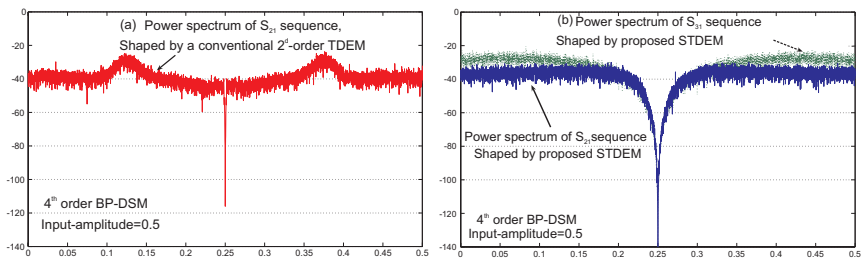

Fig. 7. $S_{21}$ in: a)the conventional TDEM's SB, b)the proposed ESB

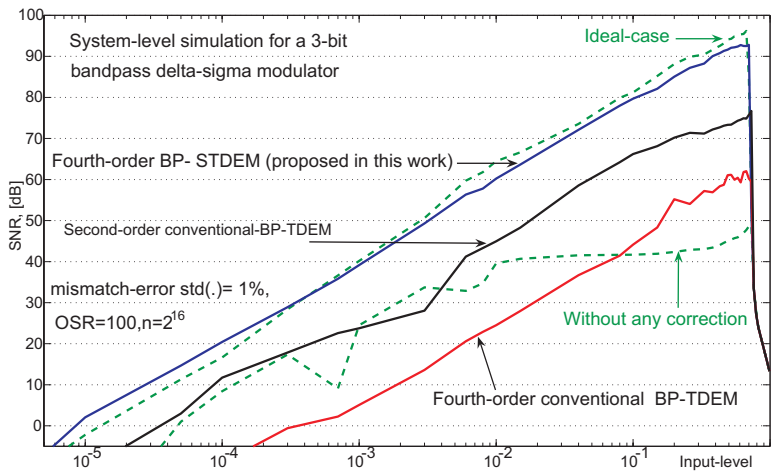

Fig. 8. Performance comparison between TDEM and STDEM algorithms

\section{CONClusion}

A hardware efficient DEM algorithm and its related circuits were presented for a high performance multibit delta-sigma modulator. These circuits use an analytically discussed and optimized decision logic for a $4^{\text {th }}$ bandpass mismatch-shaping, which were also well confirmed by system and transistor level simulations. All advantages of a conventional TDEM algorithm are maintained while its instability disadvantage is eliminated by a dynamic sequence generation in modified structure. Designed circuits need a moderate area and can be clocked faster than a comparable algorithm such as SDEM.

\section{REFERENCES}

[1] R.Scheier and G.C.Temes, Understanding Delta-sigma data converters. IEEE Press, 2005.

[2] S. Norsworthy and et.al., Delta-sigma data converters, Theory, design and simulation. IEEE Press, 97.

[3] R.Schreier and B.Zhang, "Noise-shaped multibit d/a convertor employing unit elements," Electronic letters, vol. 31/20, pp. 1712-3, 0995.

[4] I.Galton, "Spectral shaping of circuit errors in digital-to-analog converters," IEEE circuit \& systems II, vol. 44, pp. 807-8, 97.

[5] E.N.Aghdam and P.Benabes, "A new mixed stable dem algorithm for bandpass multibit delta sigma adc," P.ICECS2003, pp. 962-5.

[6] E. Aghdam and P.Benabes, "Higher order dynamic element matching by shortened tree-structure in delta-sigma modulators," P. ECCTD'05, vol. I, pp. 201-4, Sept.

[7] E.Fogleman and et.al., "An audio adc delta-sigma with 100-db peak sinad and 102-db dr using a second-order mismatch-shaping dac," IEEE J.SSC, vol. SC-36, pp. 339-48, 01.

[8] P. S. R. S.Benabid, E.Najafi Aghdam, "Cmos design of a multibit bandpass continuous-time sigma delta modulator running at $1.2 \mathrm{ghz}$," Proc. of ICCDCS.2004, vol. 1, pp. 51-5, Nov. 04.

[9] P.Benabes, P.Aldebert, and A.Yahia, "Influence of the feedback dac delay on continuous-time bandpass sigma-delta coverter," Electronics Letters, vol. 36, pp. 292-4, Feb. 00.

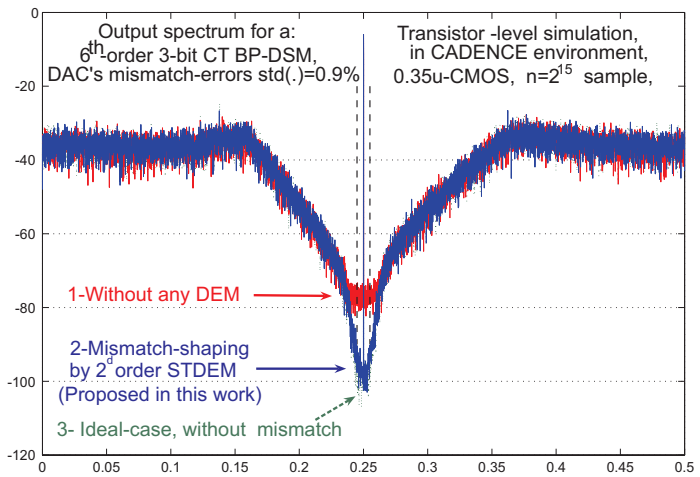

Fig. 9. Transistor-level simulation output spectrum for a 3-bit CT-DSM 